\title{
Original Research \\ Cervical balloon combined with oxytocin improves clinical efficacy and safety for induction of labor with scarred uterus
}

\author{
Dongmei $\mathrm{Ji}^{1}$, Juhua Ding ${ }^{1, *}$, Xiaoqiong $\mathrm{Li}^{1}$, Gang $\mathrm{Li}^{1}$, Dong Yin ${ }^{1}$ \\ ${ }^{1}$ Department of Obstetrics, Huai'an Maternal and Child Health Hospital, 223002 Huai'an, Jiangsu, China \\ *Correspondence: ding juh@163.com (Juhua Ding) \\ Academic Editor: Donatella Caserta \\ Submitted: 16 April 2021 Revised: 5 July 2021 Accepted: 14 July 2021 Published: 20 January 2022
}

\begin{abstract}
Background: To evaluate cervical balloon combined with oxytocin improves clinical efficacy and safety for induction of labor with scarred uterus. Methods: The clinical data of pregnant women undergoing vaginal trial delivery after cesarean section in Huai'an Maternal and Child Health Hospital, from January 2020 to January 2021 were retrospectively analyzed. Among them, 80 pregnant women with scarred uterus who were induced by cervical balloon combined with oxytocin were considered as CBO group. 80 pregnant women with natural delivery scar uterus were considered as control, who were matched to CBO group for age, gestational age, and pre-pregnancy BMI. In CBO group, Bishop score before and after induction of labor was compared. Between the CBO and control groups, mode of delivery and duration of labor were analyzed, and maternal and infant outcomes were compared. Results: The overall effective rate of cervical maturation after labor induction was $88.8 \%$, and the proportion of vaginal delivery in the CBO group were significantly higher than that in the control group. Besides, the induced labor time, first stage of labor time and the total stage of labor time in the CBO group were significantly shorter than those in the control group. There was no significant difference in maternal and infant outcomes between the two groups. Conclusion: Cervical balloon combined with oxytocin could significantly improve the clinical effect of parturients with scar uterine without increasing maternal and infant complications, which is a safe and effective strategy that can be applied in clinical practice.
\end{abstract}

Keywords: Cervical balloon; Oxytocin; Scarred uterus; Pregnancy induced labor; Complications

\section{Introduction}

Scarred uterus refers to the uterus that has undergo cesarean sections or other uterine operations. In China, the cesarean section rate is extremely high. According to the epidemiological data, the rate of cesarean section in 2016 was $40.6 \%$ [1]. With the opening of the "comprehensive two-children" policy [2], the proportion of women with scarred uterus who become pregnant again will continue to rise. Currently, the main delivery methods for re-pregnancy with scarred uterus after cesarean section include elective repeat cesarean delivery (ERCD) and trial of labor after cesarean (TOLAC) [3]. Compared to ERCD, TOLAC is costeffective and can significantly reduce the incidence of postdelivery pain and infection. However, due to the poor maturity of the cervix of scar uterus, the risk of uterine rupture is 3 times higher than that of natural labor [4]. Thus, choosing the appropriate induction way to promote the cervical maturity in pregnant women is crucial to the success of trial labor of scar uterus and vagina [5]. At present, there are various methods to promote cervical dilator balloon. For example, cervical dilated balloon has mechanical stimulation on the lower part of the uterus and cervix, which induces the release of prostaglandins in the cervix, and promotes the cervical maturation. In addition, balloon tension promotes the dissolution of cervical canal and the dilation of uterine orifice through the dilation and compression of cervical canal. The effectiveness of cervical dilated balloon has been confirmed in numerous studies [6,7]. Oxytocin induced labor is a safe, low-cost and simple method. The feasibility and effectiveness of oxytocin in the trial labor of scar uterus have been confirmed by many studies [8] and related guidelines [9,10]. The American College of Obstetricians and Gynecologists (ACOG) practice guidelines suggest that mechanical methods combined with oxytocin can also be used in scarred uterus of pregnant women [11], but few studies have reported the combination of these two methods. To further investigate the practical effect of cervical balloon combined with oxytocin in labor induction, its safety and effectiveness were evaluated by comparing the control group of uninduced VBACs.

\section{Materials and methods}

\subsection{Clinical data collection}

The present study is a retrospective study and data were collected in Huai' an Maternal and Child Health Hospital (Jiangsu Province, China) from January 2020 to January 2021. Patients $(n=80$, female) with scarred uterus received cervical balloon combined with oxytocin for induction of labor were collected and classified as CBO group. 80 cases of women who gave birth in scarred uterus with matching age, gestational age, and pre-pregnancy body mass index (BMI) during the same period were selected 
as the control group. Inclusive criteria: (1) Cephalic position singleton pregnancy, 37-41 weeks of gestation; (2) CBO group with indications of labor induction (postdate, oligohydramnios, gestational diabetes mellitus, intrahepatic cholestasis of pregnancy, gestational hypertension disease well controlled gestational hypertension, vaginal trial conditions and pregnancy termination requirements); (3) CBO group with normal non-stress test (NST) before induced labor; (4) The duration from the last cesarean section was more than 2 years, the scar healed well, and there was no scar defect in the lower uterine segment by ultrasound examination. Exclusion criteria: (1) Fetal congenital abnormalities or estimated birth weight over $4000 \mathrm{~g}$; (2) Contraindications for vaginal delivery; (3) Prenatal blood disorders, cardiopulmonary, renal or liver diseases; (4) Placenta previa, placental abruption, intrauterine infection, placenta abruptio and intrauterine infection.

\subsection{Natural delivery in the control group}

Two days before the natural childbirth, pregnant women were examined, observed and the vaginal condition were recorded. The physical and mental health of these women and the health of the fetus were recorded in detail. Based on the maturity of the cervix of the pregnant woman, the maternal score was counted to predict the problems that may occur during the delivery process so that able to prepare in advance.

\subsection{Grouping and treatment}

In the $\mathrm{CBO}$ group, the cervical dilation balloon ( $\mathrm{SCW}$ Medicath Ltd, Shenzhen, China) was placed vaginally at 9 pm the previous day. The methods were as follows: (1) Lithotomy position, performed routine disinfection of the vulva and perineum, placed the speculum into the vagina and fully exposed the cervix, and used iodophor to disinfect the vagina and cervix; (2) The anterior lip of the cervix was clamped by cervical forceps, the balloon catheter was clamped with long forceps, and the distal balloon part of the catheter was slowly placed into the uterine cavity along the lateral wall of the cervix, avoiding the placenta position; (3) Foley catheter balloon was gently inserted into the cervix with toothless oval forceps, and ensure that the balloon passes through the internal orifice of the cervix. Then, $40 \mathrm{~mL}$ of normal saline was slowly injected into the balloon, making the expanded balloon against the internal orifice of the cervix by pulling the catheter outward. Afterwards, the vaginal retractor was taken out and the water was injected gradually up to $80 \mathrm{~mL}$. Finally, the tail of balloon was fixed inner side of the thigh of the pregnant woman with adhesive tape without restricting movement.

(4) NST was performed 30 min after balloon placement, during which fetal heart rate, fetal movement and general conditions of the pregnant woman were monitored. On the next day, in case of premature rupture of membranes, regular contractions in labor, and excessive/frequent con- tractions, the balloon was removed at any time. After $12 \mathrm{~h}$ of placement of the balloon, the balloon was sterilized and taken out at about 9 am. The next day, amniotic fluid and fetal heart rate were monitored for $1 \mathrm{~h}$, oxytocin (Haitong Pharmaceutical Co., Ltd, Chengdu, China; zzy51021982, $1 \mathrm{~mL}$ : 5 units, $2.5 \mathrm{U}+0.9 \%$ normal saline $500 \mathrm{~mL}$ ) was administered intravenously. From 8 drops/min, adjust the drop speed according to the contractions (intensity, interval time and duration) of pregnant women, adjust the dropping speed once every 15-20 min, add 8 drops or 4 drops every time until the effective contractions ( 3 contractions occur in $10 \mathrm{~min}$, lasting 30-60 s), and the fastest drop speed should not exceed $20 \mathrm{mU} / \mathrm{min}$. During the process of intravenous oxytocin, midwives closely monitored the fetal heart and contractions. If a pregnant woman did not enter active delivery within $48 \mathrm{~h}$, she was considered to have failed labor induction. For instance, Elective Repeat Cesarean Section (ERCS) and Trial of Labor After Cesarean section (TOLAC). If induced labor $\geq 8 \mathrm{~h}$ still no labor should be reevaluation of whether vaginal delivery, and again with the family to explain the condition, and transfer to cesarean section if necessary. During induction of labor, the electronic fetal heart rate was monitored continuously, and the vital signs, chief complaints and general conditions of the patients were closely observed. The labor process was closely monitored. If fetal distress, abnormal labor process and threatened uterine rupture occurred during the labor process, cesarean section was immediately performed. The vital signs, vaginal bleeding and uterine involution were closely observed after delivery.

\subsection{Effectiveness evaluation}

Evaluation of cervical ripening effect: Bishop score [12] was used before and $12 \mathrm{~h}$ after induction of labor. The Bishop score is an international standard for evaluating the maturity of the cervix. A higher score means a more mature cervix and a higher success rate for induction of labor [13]. The total score is based on a number of factors other than the texture of the cervix, such as the position of the cervix, the height of exposure, the length of the cervix and the degree of dilation of the cervix. Two score increase $>2$ points means significant effective, 2 points means effective, $<2$ points means invalid, overall effective $=$ significant effective + effective.

Evaluation of mode of delivery and duration of labor: vaginal delivery rate was compared, i.e., (spontaneous vaginal delivery + forceps delivery cases)/80. In this study, 80 cases of pregnant women with scar uterus induced by cervical balloon combined with oxytocin were selected as the experimental group, and 80 cases of pregnant women with scar uterus induced by natural delivery matched with age, gestational age and pre-pregnancy Body Mass Index (BMI) at 1:1 were selected as the normal control. The evaluation of labor process time includes induced labor time, first labor process time and total labor process time; induced labor 
Table 1. Comparison of two groups of general data $(\bar{x} \pm \boldsymbol{s})$.

\begin{tabular}{lccc}
\hline & Control group & CBO group & $p$ values \\
\hline Number of cases & 80 & 80 & \\
Age (years) & $28.15 \pm 4.51$ & $27.69 \pm 4.49$ & $0.517^{a}$ \\
BMI $\left(\mathrm{kg} / \mathrm{m}^{2}\right)$ & $20.53 \pm 3.32$ & $21.30 \pm 3.60$ & $0.163^{a}$ \\
Gestational age (weeks) & $39.01 \pm 1.00$ & $38.94 \pm 1.01$ & $0.638^{a}$ \\
Newborn birth weight $(\mathrm{g})$ & $3640.06 \pm 308.45$ & $3570.52 \pm 327.78$ & $0.169^{a}$ \\
\hline Note: ${ }^{a}$ stands for independent $t$-test.
\end{tabular}

Note: ${ }^{a}$ stands for independent $t$-test.

time refers to the time from induced labor to normal uterine contraction; first labor process time refers to the time from regular uterine contraction to complete opening of uterine orifice; total labor process time refers to the time from normal uterine contraction to placenta delivery.

\subsection{Safety evaluation}

The incidence of uterine rupture was compared, and the amount of postpartum hemorrhage, neonatal weight, and Apgar score (5 min) (Apgar score 7 to 10 for normal neonates, 4 to 7 for mild asphyxia, and 0 to 4 for severe asphyxia) were recorded. The occurrence of maternal complications (postpartum hemorrhage, postpartum fever, puerperal infection) and adverse reactions (nausea, vomiting, fever) were analyzed.

\subsection{Statistical analysis}

SPSS 25.0 software (IBM corp, NY, USA) was used for data analysis. The measurement data of normal distribution were expressed as mean \pm standard deviation. The measurement data of non-normal distribution were expressed by median and interquartile Distance $\mathrm{M}\left(\mathrm{P}_{25}-\mathrm{P}_{75}\right)$, and the rank sum test was used for the comparison between groups; the count data were described by frequency and percentage $(\%)$, and the comparison between groups was performed by $\chi^{2}$ test or Fisher test, and the test level $\alpha=0.05$, with $p<0.05$ as the difference was statistically significant.

\section{Results}

\subsection{Comparison of general data between the two groups}

There were no significant differences in maternal age, delivery gestational weeks, body mass index (BMI), gestational age and neonatal birth weight between the two groups $(p>0.05)$, as shown in Table 1 .

\subsection{Comparison of cervical ripening effect in $\mathrm{CBO}$ group}

Before and after $12 \mathrm{~h}$ of induction of labor, the median Bishop score was 4.0 and 7.0 respectively in the CBO group $(Z=-11.253, p<0.05)$. The overall effective rate of promoting cervical maturity after induction was $88.8 \%$, as shown in Table 2.

\subsection{Comparison of mode of delivery and duration of labor} between the two groups

The total vaginal delivery rate of the $\mathrm{CBO}$ group was $93.7 \%$, which was higher than that of the control group $(77.5 \%)$, and the difference was statistically significant $(p$ $<0.05)$. The induction time of labor in the $\mathrm{CBO}$ group was $13(9,16) \mathrm{h}$, the first stage of labor was $5(4,5) \mathrm{h}$, and the total stage of labor was $8(8,9.75) \mathrm{h}$, which was significantly shorter than that in the control group $[21(18,23) \mathrm{h}$, $8(6,9) \mathrm{h}, 15(13,20) \mathrm{h}$ ], and the difference was statistically significant $(p<0.05)$, which was shown in Table 3.

\subsection{Comparison of maternal and infant outcomes between the two groups}

There was no uterine rupture in the two groups. $2 \mathrm{~h}$ after delivery, the blood loss in the CBO group and the control group was $(300.53 \pm 61.65) \mathrm{mL}$ and $(295.44 \pm 58.31) \mathrm{mL}$, respectively. The number of newborns with Apgar score $>7$ in the CBO group and the control group was $80(100 \%)$ and $77(96.3 \%)$, respectively. There was no statistical significance in the incidence of maternal complications (postpartum massive hemorrhage, postpartum fever, puerperal infection) and adverse reactions (nausea and vomiting) between the two groups $(p>0.05)$, as shown in Table 4 .

\section{Discussion}

\subsection{Cervical balloon combined with oxytocin can further promote cervical ripening}

Cervical ripening is the decisive factor for successful induction of labor [14]. Cervical maturation is the early part of labor, due to the increase of cellular matrix metalloproteinases and decreased content of extracellular matrix protein, the emergence of tissue compliance gradually increased, cervical length gradually shortened. The essence is the cervical collagen fiber morphology and character change. At the same time, the content of hyaluronic acid in the cervix increases and hydration destroys the collagen fiber network, causing cervical ripening and releasing oxytocin, which can promote uterine contraction $[15,16]$. Low cervical maturity in late pregnancy is one of the direct causes of insufficient uterine contractility. Promoting cervical maturity can reduce the risk of uterine rupture and cervical laceration. A large sample study showed that among women with a history of vaginal delivery, TOLAC had a $91 \%$ success rate when the cervix was ripe, but a $77 \%$ suc- 
Table 2. Comparison of the effect of cervical maturation before and after labor induction.

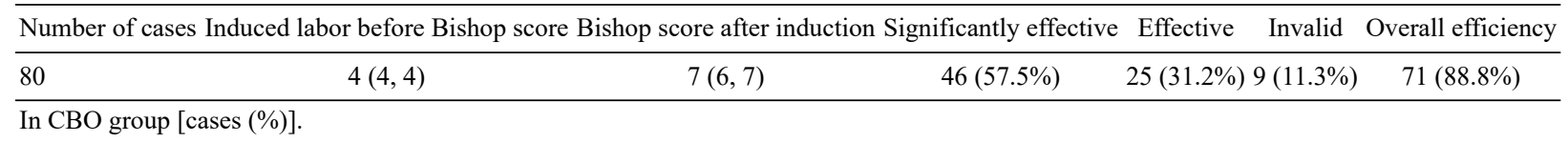

Table 3. Comparison of modes of delivery and duration of labor [cases (\%) $\left./ \bar{x} \pm \mathbf{s} / \mathbf{M}\left(\mathbf{P}_{25}-\mathbf{P}_{75}\right)\right]$.

\begin{tabular}{lccc}
\multicolumn{4}{c}{ labor [cases $\left.(\mathbf{\%}) / \bar{x} \pm \mathbf{s} / \mathbf{M}\left(\mathbf{P}_{25}-\mathbf{P}_{75}\right)\right]}$. \\
\hline & Control group & CBO group & $p$ values \\
\hline Number of cases & 80 & 80 \\
Vaginal natural delivery & $62(77.5 \%)$ & $75(93.7 \%)$ & $0.003^{a}$ \\
Cesarean delivery & $18(22.5 \%)$ & $5(6.3 \%)$ & \\
Induce labor time (h) & $21(18,23)$ & $13(9,16)$ & $0.000^{b}$ \\
First stage of labor time (h) & $8(6,9)$ & $5(4,5)$ & $0.000^{b}$ \\
Total duration of labor (h) & $15(13,20)$ & $8(8,9.75)$ & $0.000^{b}$ \\
\hline Note: ${ }^{a}$ represents $\chi^{2}$ test, $^{b}$ represents rank sum test.
\end{tabular}

Note: ${ }^{a}$ represents $\chi^{2}$ test, ${ }^{b}$ represents rank sum test.

cess rate when the cervix was immature [17]. Therefore, it is very important to adopt the induction that can rapidly promote the cervical maturation of women before parturient to improve the success rate of Vaginal Birth After Cesarean Section (VBAC) for women with scarred uterus. This study found that cervical balloon combined with oxytocin induction of labor could promote cervical ripening of women with scarred uterus. Compared with before induction of labor, and the overall effective rate of cervical ripening was also higher., and the overall effective rate of cervical ripening was higher. Using the "bionic principle", the cervical dilation balloon not only promotes cervical ripening by mechanical stimulation, but also regulates the synthesis and secretion of hormones such as prostaglandins, improves cervical physiological function and increase the intensity and frequency of uterine contraction reaction. In addition, oxytocin can combine with uterine smooth muscle receptor to cause uterine contractions and promote delivery, it also can stimulate the production of prostaglandins in decidual tissue and facilitates cervical ripening.

\subsection{Cervical balloon combined with oxytocin can shorten the labor process}

Stock found [18] that compared with expected management, pregnant women with scarred uterus at 39-41 weeks of pregnancy may reduce the chance of scarring the uterus again. In this study, the vaginal delivery rate of the cervical balloon combined with oxytocin group was $93.7 \%$, which was significantly higher than $77.5 \%$ in the control group. However, the cesarean section rate of the cervical balloon combined with oxytocin group $6.3 \%$, which was significantly lower than $22.5 \%$ in the control group, suggesting that the cervical balloon combined with oxytocin could promote cervical ripening and improve the vaginal delivery rate. In addition, using cervical balloon combined with oxytocin for induction of labor could effectively shorten the time of labor process, since the median of induced labor was $13 \mathrm{~h}$, the median of the first stage of labor was $5 \mathrm{~h}$, the median of total labor was 8 hours, which was significantly shorter than that of the control group. The reason may be that cervical dilatation balloon promotes cervical ripening, combined with oxytocin to stimulate uterine contraction, the joint effect of the two can accelerate the process of labor. Studies have shown that more than $90 \%$ of uterine rupture occurs in the first stage of labor in TOLAC, and the highest incidence of uterine rupture occurs when the uterine orifice is $4-5 \mathrm{~cm}$ wide [5], suggesting that the first stage of labor is the point to the scarred uterus. Some scholars have shown that $[19,20]$, cervical balloon induction method is especially conducive to avoid prolonged contractions, such as placental insufficiency, fetal intrauterine growth restriction, oligohydramnios and scar uterus. Compared with the spontaneous vaginal delivery, the advantages of cervical balloon combined with oxytocin in shortening the delivery cycle is more obvious.

\subsection{Cervical balloon combined with oxytocin is safe}

Scarred uterus for hasten parturition, induction of labor is the greatest risk of threatened uterine rupture or uterine rupture. The thickness of the lower uterine scar is directly related to the risk of uterine rupture. Due to the lack of elasticity of the scar, the weak part of the scar is prone to rupture as intrauterine pressure increases during labor.

A systematic review [21] showed a specificity of $91 \%$ and a sensitivity of $61 \%$ for predicting uterine rupture during TOLAC when the thickness of uterine scar ranged from $2.0 \mathrm{~mm}$ to $3.0 \mathrm{~mm}$. In this study, no uterine rupture occurred in either natural delivery or cervical balloon combined with oxytocin induced labor. The uterine contractions caused by the cervical balloon are weaker and less stimulating to the uterus, and the use of combined oxytocin also prevent the balloon from causing regular contractions and ensure the smooth implementation of labor induction. A multicenter study showed [22] that the incidence of mechanical induced uterine rupture was the lowest. Foreign scholars Palatnik et al. [23] and Dekker et al. [24] also confirmed the effectiveness of oxytocin in 1538 cases and 1008 cases of scarred uterus. The results showed that the vaginal delivery rate was $92.2 \%$, and the incidence of uterine rupture was only $0.09 \%$. This study excluded obstetric complications such as placenta previa and placental abruption that were not suitable for vaginal delivery. Postpartum Hemorrhage $(\mathrm{PH})$, as one of the main risks in the process of scar uterine and vaginal trial delivery, is mainly related to lower uterine scar and uterine rupture. In addition, the placenta is easy to adhere or implant scar uterine cavity wall, resulting in postpartum unable to discharge by itself, affecting 
Table 4. Comparison of maternal and infant outcomes between the two groups [cases $(\%) / \bar{x} \pm \mathrm{SD}]$.

\begin{tabular}{lccc}
\hline & Control group & CBO group & $p$ values \\
\hline Number of cases & 80 & 80 & \\
Blood loss 2 h later & $295.44 \pm 58.31$ & $300.53 \pm 61.65$ & $0.593^{b}$ \\
Apgar score & $9(8,10)$ & $9(8,10)$ & $0.632^{c}$ \\
Apgar classification & & & \\
$\quad$ Severe asphyxia & $0(0.0 \%)$ & $0(0.0 \%)$ & $0.245^{d}$ \\
$\quad$ Mild choking & $3(3.7 \%)$ & $0(0.0 \%)$ & \\
$\quad$ Normal & $77(96.3 \%)$ & $80(100 \%)$ & \\
Adverse events & & & \\
$\quad$ Nausea & $14(17.5 \%)$ & $12(15 \%)$ & $0.668^{a}$ \\
$\quad$ Vomiting & $10(12.5 \%)$ & $7(8.8 \%)$ & $0.442^{a}$ \\
Maternal complications & & & \\
$\quad$ Postpartum hemorrhea & $0(0.0 \%)$ & $0(0.0 \%)$ & \\
$\quad \begin{array}{l}\text { Postpartum fever } \\
\quad \text { Puerperal infection }\end{array}$ & $5(6.3 \%)$ & $2(2.5 \%)$ & $0.443^{d}$ \\
\hline Note: ${ }^{a}$ represents $\chi^{2}$ test, ${ }^{b}$ represents independent $t$-test, ${ }^{c}$ represents rank \\
sum test, and ${ }^{d}$ represents Fisher test.
\end{tabular}

uterine contraction and resulting in massive bleeding. In this study, balloon cervix maturation combined with oxytocin enhancement of uterine contraction did not increase the risk of $\mathrm{PH}$. In this study, a low incidence of bleeding was expected in the collective of patients with a history of scarred uterus. There was no increase in the incidence of perinatal complications such as postpartum fever, puerperal infection, nausea, vomiting and fever. There was no difference in Apgar score and incidence of neonatal asphyxia, indicating no significant difference in pregnancy outcome between the two groups. The combination of cervical balloon dilation with oxytocin did not increase adverse events or adverse pregnancy reactions. It is evident that it is safe and worthy of clinical promotion.

\section{Conclusions}

Compared with traditional natural delivery, cervical balloon combined with oxytocin can shorten the labor process, increase the vaginal delivery rate, and do not increase the risk of postpartum hemorrhage and infectious complications. It is safe and effective for scarred uterus and pregnant women, and is worth popularizing and applying.

\section{Abbreviations}

ERCD, Elective Repeat Cesarean Delivery; TOLAC, Trial of Labor After Cesarean; ACOG, American College of Obstetricians and Gynecologists; NST, non-stress test; VBAC, Vaginal Birth After Cesarean Section.

\section{Author contributions}

DJ and JD designed the study, supervised the data collection, XL analyzed the data, interpreted the data, GL and DY prepare the manuscript for publication and reviewed the draft of the manuscript. All authors have read and approved the manuscript.

\section{Ethics approval and consent to participate}

All subjects gave their informed consent for inclusion before they participated in the study. Ethical approval was obtained from the Ethics Committee of Huai'an Maternal and Child Health Hospital, Jiangsu Province (Approval No. 2019058).

\section{Acknowledgment}

Thanks to all the peer reviewers for their opinions and suggestions.

\section{Funding}

This research received no external funding.

\section{Conflict of interest}

The authors declare no conflict of interest.

\section{Statement of informed consent}

Written informed consent was obtained from a legally authorized representative(s) for anonymized patient information to be published in this article.

\section{References}

[1] Liu X, Huang D, Landon MB, Cheng W, Chen Y. Trends in Cesarean Delivery Rate after Cessation of the One-Child Policy in China. American Journal of Perinatology. 2020. (in press)

[2] Selin L, Wennerholm U, Jonsson M, Dencker A, Wallin G, Wiberg-Itzel E, et al. High-dose versus low-dose of oxytocin for labour augmentation: a randomised controlled trial. Women and Birth. 2019; 32: 356-363.

[3] ACOG Practice Bulletin No. 205: Vaginal Birth After Cesarean Delivery. Obstetrics \& Gynecology. 2019; 133: e110-e127.

[4] Macones GA, Peipert J, Nelson DB, Odibo A, Stevens EJ, Stamilio DM, et al. Maternal complications with vaginal birth after cesarean delivery: a multicenter study. American Journal of Obstetrics and Gynecology. 2005; 193: 1656-1662. 
[5] Holmgren C, Scott JR, Porter TF, Esplin MS, Bardsley T. Uterine Rupture with Attempted Vaginal Birth after Cesarean Delivery: decision-to-delivery time and neonatal outcome. Obstetric Anesthesia Digest. 2012; 33: 109-110.

[6] Kehl S, Weiss C, Wamsler M, Beyer J, Dammer U, Heimrich J, et al. Double-balloon catheter and sequential vaginal prostaglandin E2 versus vaginal prostaglandin E2 alone for induction of labor after previous cesarean section. Archives of Gynecology and Obstetrics. 2016; 293: 757-765.

[7] Manish P, Rathore S, Benjamin SJ, Abraham A, Jeyaseelan V, Mathews JE. A randomised controlled trial comparing $30 \mathrm{~mL}$ and $80 \mathrm{~mL}$ in Foley catheter for induction of labour after previous Caesarean section. Tropical Doctor. 2016; 46: 205-211.

[8] Penfield CA, Wing DA. Labor Induction Techniques: Which Is the Best? Obstetrics and Gynecology Clinics of North America. 2017; 44: 567-582.

[9] Grobman W. Practice Bulletin no. 184: Vaginal Birth after Cesarean Delivery. Obstetrics and Gynecology. 2017; 130: e217e233.

[10] Martel M, MacKinnon CJ. No. 155-Guidelines for Vaginal Birth after Previous Caesarean Birth. Journal of Obstetrics and Gynaecology Canada. 2018; 40: e195-e207.

[11] ACOG Practice bulletin no. 115: Vaginal birth after previous cesarean delivery. Obstetrics \& Gynecology. 2010; 116: 450463.

[12] Miller NR, Cypher RL, Foglia LM, Pates JA, Nielsen PE. Elective Induction of Labor Compared with Expectant Management of Nulliparous Women at 39 Weeks of Gestation: a Randomized Controlled Trial. Obstetrics and Gynecology. 2015; 126: 1258 1264.

[13] Grange J, Dimet J, Vital M, Le Thuaut A, Ducarme G. Doubleballoon catheter compared to vaginal dinoprostone for cervical ripening in obese women at term. Gynecologie, Obstetrique, Fertilite \& Senologie. 2017; 45: 521-527.

[14] Al-Ibraheemi Z, Brustman L, Bimson BE, Porat N, Rosenn B. Misoprostol with Foley Bulb Compared with Misoprostol alone for Cervical Ripening: a Randomized Controlled Trial. Obstetrics and Gynecology. 2018; 131: 23-29.
[15] Gonzalez JM, Franzke C, Yang F, Romero R, Girardi G. Complement activation triggers metalloproteinases release inducing cervical remodeling and preterm birth in mice. American Journal of Pathology. 2011; 179: 838-849.

[16] Mahendroo M. Cervical remodeling in term and preterm birth: insights from an animal model. Reproduction. 2012; 143: 429438.

[17] Grobman WA, Gilbert S, Landon MB, Spong CY, Leveno KJ, Rouse DJ, et al. Outcomes of induction of labor after one prior cesarean. Obstetrics and Gynecology. 2007; 109: 262-269.

[18] Stock SJ, Ferguson E, Duffy A, Ford I, Chalmers J, Norman JE. Outcomes of induction of labour in women with previous caesarean delivery: a retrospective cohort study using a population database. PLoS ONE. 2013; 8: e60404.

[19] Miller TD, Davis G. Use of the Atad catheter for the induction of labour in women who have had a previous Caesarean section-a case series. Australian \& New Zealand Journal of Obstetrics \& Gynaecology. 2005; 45: 325-327.

[20] Khotaba S, Volfson M, Tarazova L, Odeh M, Barenboym R, Fait $\mathrm{V}$, et al. Induction of labor in women with previous cesarean section using the double balloon device. Acta Obstetricia et Gynecologica Scandinavica. 2001; 80: 1041-1042.

[21] Kok N, Wiersma IC, Opmeer BC, de Graaf IM, Mol BW, Pajkrt E. Sonographic measurement of lower uterine segment thickness to predict uterine rupture during a trial of labor in women with previous Cesarean section: a meta-analysis. Ultrasound in Obstetrics \& Gynecology. 2013; 42: 132-139.

[22] Sheibani L, Wing DA. A safety review of medications used for labour induction. Expert Opinion on Drug Safety. 2018; 17: 161-167.

[23] Palatnik A, Grobman WA. Induction of labor versus expectant management for women with a prior cesarean delivery. American Journal of Obstetrics and Gynecology. 2015; 212: 358.e1358.e6.

[24] Dekker G, Chan A, Luke C, Priest K, Riley M, Halliday J, et al. Risk of uterine rupture in Australian women attempting vaginal birth after one prior caesarean section: a retrospective population-based cohort study. BJOG: an International Journal of Obstetrics \& Gynaecology. 2010; 117: 1358-1365. 